\title{
Fractal Chemical Kinetics: Reacting Random Walkers ${ }^{1}$
}

\author{
L. W. Anacker, ${ }^{2}$ R. Kopelman, ${ }^{2}$ and J. S. Newhouse ${ }^{2}$
}

Computer simulations on binary reactions of random walkers $(A+A \rightarrow A)$ on fractal spaces bear out a recent conjecture: $\left(\rho^{-1}-\rho_{0}^{-1}\right) \propto t^{f}$, where $\rho$ is the instantaneous walker density and $\rho_{0}$ the initial one, and $f=d_{s} / 2$, where $d_{s}$ is the spectral dimension. For the Sierpinski gaskets: $d=2,2 f=1.38\left(d_{s}=1.365\right)$; $d=3,2 f=1.56\left(d_{s}=1.547\right)$; biased initial random distributions are compared to unbiased ones. For site percolation: $d=2, p=0.60,2 f=1.35\left(d_{s}=1.35\right)$; $d=3, p=0.32,2 f=1.37\left(d_{s}=1.4\right)$; fractal-to-Euclidean crossovers are also observed. For energetically disordered lattices, the effective $2 f$ (from reacting walkers) and $d_{s}$ (from single walkers) are in good agreement, in both two and three dimensions.

KEY WORDS: Fractal; reacting random walkers; spectral dimension; Sierpinski gasket; percolation; energy disorder.

\section{INTRODUCTION}

Within the last year, intrinsic dynamical properties of fractals, e.g., random walk, conductivity, photon and magnon effects, have been related to an unexpected new dimension $d_{s}$, the spectral (fracton) dimension. ${ }^{(1-4)}$ Specifically, for $t \rightarrow \infty$, the mean number of distinct sites visited by a random walker is $\langle S(t)\rangle \propto t^{f}$, where $d_{s}=2 f \leqslant 2$. For fractal spaces the fact that $f<1$ (e.g., $f=2 / 3$ ) leads to drastic deviations from the classical laws involving the diffusion constant, the phonon density of states and the conductivity. It has also been conjectured that the diffusion-limited binary reaction coefficient is related to the same spectral dimension. ${ }^{(5)}$ Specifically, it was suggested that for one kind of reactant:

$$
A+A \rightarrow \text { products }
$$

\footnotetext{
${ }^{1}$ Supported by NSF Grant No. DMR 8303919.

${ }^{2}$ Department of Chemistry, University of Michigan, Ann Arbor, Michigan 48109.
} 
where the $A$ species consists of random walkers, the integrated rate law is (for long times):

$$
\rho^{-1}-\rho_{0}^{-1} \propto t^{f}, \quad f=d_{s} / 2 \leqslant 1
$$

where $\rho$ is the walker density at time $t$ and $\rho_{0} \equiv \rho(t=0)$. The concomitant differential rate law is

$$
-\frac{d \rho}{d t} \propto \rho^{2} t^{f-1}, \quad t>0
$$

The above equations should be contrasted with the classical (Euclidean space) equations

$$
\begin{array}{r}
\rho^{-1}-\rho_{0}^{-1} \propto t \\
-\frac{d \rho}{d t} \propto \rho^{2}
\end{array}
$$

A more complicated situation exists for the reactions $A+B \rightarrow$ products, where $B$ may be a sitter (trap) or walker, and conflicting results have very recently been claimed. ${ }^{(6-8)}$ The fluctuation problem, involving the "two-kind" reactions $(A+B)$, is an old one and also arises in Euclidean spaces. ${ }^{(9-14)}$ For simplicity, we concentrated on the "one kind" reactions $(A+A)$. This has been both our experimental and theoretical approach. ${ }^{(5,15)}$

We tested Eqs. (2) abd (3) by performing Monte Carlo simulations on (a) deterministic fractals, the two- and three-dimensional Sierpinski gaskets; (b) random fractals, the two- and three-dimensional percolating clusters (square and simple cubic); and c) possible effective fractals, the two- and three-dimensional energetically disordered lattices where the waiting times include Boltzmann factors. Only nearest-neighbor steps were allowed. If two walkers are simultaneously on the same site, then

$$
A+A \rightarrow A
$$

We found surprisingly good agreement between $f^{\prime}$, the empirical exponent, and $f=d_{s} / 2$, the conjectured ${ }^{(5)}$ result, for both Sierpinski gaskets and incipient percolating clusters, for both two- and three-dimensional topologies. Furthermore, the same asymptotic result was found for a biased, rather than a uniformly random, initial $\rho_{0}$ on a Sierpinski gasket. Moreover, for infinite clusters well above the percolation threshold, the reacting walker problem parallels the single walker behavior: A crossover from fractal-toEuclidean law, associated with a correlation length and a crossover time, 
occurs. ${ }^{(16-20)}$ Even the energetically disordered lattice shows good agreement between $f^{\prime}(T)$ and $f(T)$, where $f(T)$ is the single random walk effective fractal exponent ${ }^{(21)}$ for the given temperature $(T)$ :

$$
\langle S(t)\rangle \propto t^{f(T)}
$$

and $S(t)$ is the number of distinct sites visited at time $t$.

\section{METHODS}

\subsection{Sierpinski Gasket}

Reacting random walker simulations were performed on an eight-order planar two-dimensional (2d) Sierpinski gasket and on a six-order threedimensional (3d) Sierpinski gasket. In both cases, the gaskets were generated iteratively and consisted of approximately 10000 sites (vertices). At each step, a walker must move with equal probability to one of its nearestneighbor sites. Walkers moving to one of the vertices of the largest triangle are not allowed to leave the finite gasket, but are given an equal probability of moving to one of the two (2d) or three (3d) possible nearest-neighbor sites. The direction in which each walker moves is decided by the uniformly distributed pseudo-random number generator FUNIF. ${ }^{(22)}$

Walkers were randomly place on the two- and three-dimensional gaskets in an unbiased manner (equal probability of landing on any unoccupied site) and also in a given biased manner on the planar gasket. Specifically; the biased placement of walkers preferentially placed them near the top of the gasket: the walker is assigned an equal probability of landing on any of the gaskets' $L_{\text {total }}$ layers (top to bottom); subsequent placement of the walker in the $L_{i}$ layer uses a conditional probability, $p\left(L_{i}\right)$ :

$$
p\left(L_{i}\right) \equiv \begin{cases}L_{i}^{-1}, & \text { for } 2 \mathrm{~d} \\ 2 / L_{i}\left(L_{i}+1\right), & \text { for } 3 \mathrm{~d}\end{cases}
$$

If the walker does not land on a vacant site, a new set of random numbers is used to select a layer and position within that layer.

Simulations for the unbiased placement of walkers started with initial walker densities of $\rho_{0}=0.1$ and were followed for 4000 steps. These conditions were used in both two and three dimensions with 500 runs in each dimension. Simulations which included a bias in initial walker placement consisted of 3000 runs and were followed for 2000 steps. With the biased placement, the initial local density of walkers near the top of the gasket is greater than at the bottom. The average initial density was 0.02 . 


\subsection{Binary Lattices}

A uniformly distributed pseudo-random number generator ${ }^{(23)}$ is used to generate both two and three-dimensional binary lattices. Random numbers from separate uniform distributions are then used to initially place the walkers and to determine the direction in which a surviving walker moves at each step. Reacting random walker simulations in two dimensions were performed on $200 \times 200$ square lattices and in three dimensions on $40 \times 40 \times 40$ cubic lattices. Periodic boundary conditions were imposed, and walkers were started on sites in the largest cluster with a probability of 0.02 which gives $\left\langle\rho_{0}\right\rangle \cong 0.02$. At each step a walker was forced to move to one of its nearest-neighbor open sites. This method of "forced walk" is analogous to the "myopic ant" method. (24)

Two-dimensional binary lattices were generated with site probabilities of $p=0.40,0.60$, and 0.80 (compared with the critical percolation probability $p_{c}=0.593$ ) and three-dimensional lattices with $p=0.32,0.40$, and 1.00 (compared with $p_{c}=0.311$ ). Walks were followed for 2000 moves and 100 lattice realizations were performed for each of the above lattices. Simulations on $100 \times 100$ square lattices with $p=0.40<p_{c}$ included walks on the 50 largest clusters. The initial condition on this lattice was $\rho_{0}=1.0$ and walkers were followed for 1000 steps.

\subsection{Energetically Disordered Lattices}

Lattices with energetic disorder (described in greater detail in Ref. 21) were generated as the walker moved on both square and simple cubic lattices. Site energies were assigned using the uniform pseudo-random number generator FUNIF ${ }^{(22)}$ and correspond to a normalized distribution of bandwidth $W$. A random number from a separate uniform distribution was used in moving each of the surviving random walkers. On these lattices the microscopic transfer rates are asymmetric: the probability of a move to a site of higher energy includes a Boltzmann weighting factor; the probability of a move to a site of equal or lower energy is $z^{-1}$ where $z$ is the coordination number. Thus residual probability of the walker remaining on the same site for two consecutive steps is included for a site with at least one higherenergy nearest-neighbor site.

The parameter of interest on the energetically disordered lattices is the reduced temperature $T^{\prime \prime} \equiv k T / W$. In two dimensions, reduced temperatures of $T^{\prime \prime}=0.1$ and 0.2 and the temperature-independent perfect lattice were simulated. We imposed periodic boundary conditions with $\rho_{0}=0.002$ on $256 \times 256$ square lattices $\left(T^{\prime \prime}=0.2\right)$ and on $500 \times 500$ square lattices $\left(T^{\prime \prime}=0.1\right.$ and the perfect lattice). In two dimensions, 12 runs were 
performed on the perfect lattice (5000 steps), 30 runs at $T^{\prime \prime}=0.2(4000$ steps), and 52 runs at $T^{\prime \prime}=0.1$ (5000 steps). In three dimensions, ten runs were performed on $25 \times 25 \times 25$ cubic lattices with periodic boundary conditions, for each of the reduced temperatures $T^{\prime \prime}=0.03,0.05$, and 0.1 . An initial walker density of $\rho_{0}=0.1$ was used and walkers were followed for 2000 steps.

\section{RESULTS AND DISCUSSION}

\subsection{Sierpinski Gasket}

The Sierpinski gaskets represent simple geometric structures with known ${ }^{(4,25)}$ fractal dimensions, $d_{f}=\ln (d+1) / \ln 2$, and spectral (fracton) dimensions, $d_{s}=2 \ln (d+1) / \ln (d+3)$ where $\mathrm{d}$ is the Euclidean dimension. Single random walker simulations on these gaskets in both two and three dimensions have been performed ${ }^{(25)}$ and verify that the mean number of distinct sites visited in an $N$ step walk follows the asymptotic $(t \rightarrow \infty)$ relation,

$$
\left\langle S_{N}\right\rangle=a N^{f}, \quad f=d_{s} / 2
$$

reasonably well at all times. In the asymptotic limit, all our reacting walker simulations on Sierpinski gaskets satisfy the relation [compare Eq. (2)]:

$$
\left\langle\rho^{-1}-\rho_{0}^{-1}\right\rangle=a^{\prime} t^{f^{\prime}}, \quad f^{\prime} \cong d_{s} / 2
$$

In two dimensions, we obtain values of $f^{\prime}=0.68$ and 0.69 , respectively, for the biased and unbiased initial walker placements. These values are in good agreement with the theoretical (analytical) values of $d_{s} / 2=0.683$; a similar result is obtained in three dimensions where the asymptotic value of $f^{\prime}=0.78$ compares well with the theoretical $d_{s} / 2=0.774$. Our simulations corresponding to a uniformly random (unbiased) initial placement of walkers in both two and three dimensions follow the relationship in Eq. (7) quite well over the entire time range, giving values of $f^{\prime}=0.70(2 \mathrm{~d})$ and $f^{\prime}=0.78(3 \mathrm{~d})$. These two curves are surprisingly straight over the entire time range (though there is a slightly steeper slope at early times), as is shown for the top (3d) and bottom (2d) lines in Fig. 1. The higher curve in Fig. 2, which joins the lower curve for the planar Sierpinski gasket at long times, was obtained from our simulations which started the walkers with a higher local density near the top of the gasket (the density increases from bottom to top of gasket). Results of a nonlinear regression analysis on our simulation data are presented in Table I. Note that not only the exponents but also the preexponents are in good agreement with the single walker simulation 


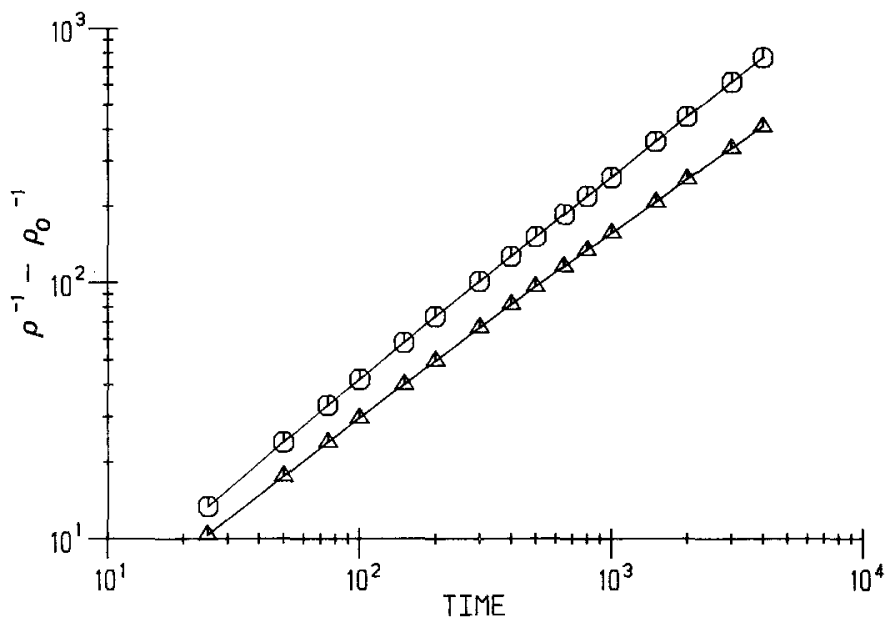

Fig. 1. $\log \left\langle\rho^{-1}-\rho_{0}^{-1}\right\rangle$ vs. $\log t$ for Sierpinski gaskets in two dimensions (bottom curve) and three dimensions (top curve) with averages of 500 runs for each curve. Walkers were randomly placed on the gasket $\left(\rho_{0}=0.1\right)$ in an unbiased manner with an equal probability of landing on any unoccupied site.

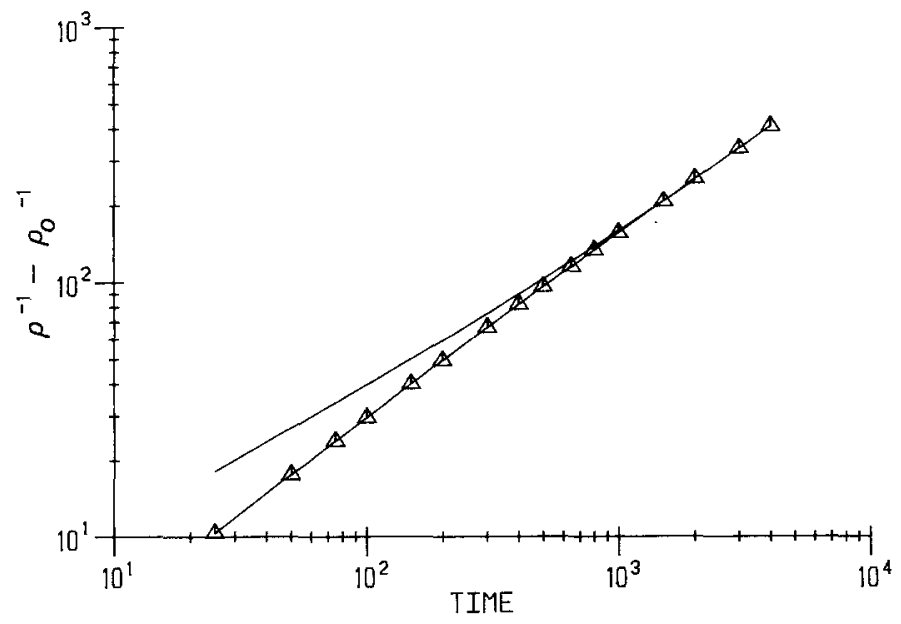

Fig. 2. $\log \left\langle\rho^{-1}-\rho_{0}^{-1}\right\rangle$ vs. $\log t$ for a planar (2d) Sierpinski gasket with biased (top curve) and unbiased (bottom curve-same as the bottom curve in Fig. 1) placement of the walkers. Averages for 3000 runs are shown in the top curve with $\rho_{0}=0.02$. 
Table I. Sierpinski Gasket Fractal Exponents, $f^{\prime}$, and Preexponents, a

\begin{tabular}{llll}
\hline & $3 \mathrm{~d}$ & \multicolumn{2}{c}{$2 \mathrm{~d}$} \\
\cline { 3 - 4 } & & Unbiased & Biased \\
\hline Reacting walkers & & & \\
$f^{\prime}$ & 0.78 & 0.69 & 0.68 \\
$a^{\prime}$ & 1.2 & 1.3 & 1.4 \\
Single walker & & & \\
$f$ & $0.753^{a}$ & $0.686^{a, b}$ & \\
$a$ & $1.318^{a}$ & $1.282^{a}$ & \\
$d_{s / 2}$ & 0.7737 & & 0.6826 \\
\hline
\end{tabular}

${ }^{a}$ Blumen et al. ${ }^{(25)}$

${ }^{b}$ Angles D'Auriac et al. ${ }^{(26)}$ give 0.682 .

results. ${ }^{(25,26)}$ We note that the biased reaction starts with a higher reaction rate, but at long times joins the unbiased case. This early time behavior is reasonable in light of the higher initial densities and is an extremely interesting case since it shows how a large perturbation (from a uniform random initial distribution) can be "overcome" within a relatively short time.

\subsection{Binary Lattices}

For binary lattices near criticality, Eq. (6) holds ${ }^{(18,21)}$ for the single walker in the asymptotic limit. Here we investigate the reacting random walker problem on square and simple cubic binary lattices over a range of open site probabilities. The simulation results are shown in the log-log plots of Figs. 3 and 4 . In our reacting random walker simulations on lattices just above criticality, $p=0.60(2 \mathrm{~d})$ and $p=0.32$ (3d), we obtain effective spectral dimensions of 1.35 (2d) and 1.37 (3d) in the asymptotic limit, compared with the spectral dimension, $d_{s} \cong 1.33$, at criticality. These results are remarkably consistent over the entire time scale (steps $10 \rightarrow 2000$ ), where we obtain effective spectral dimensions of 1.35 (2d) and 1.34 (3d). Simulations on the three-dimensional perfect lattice, $p=1.0$, gives an effective spectral dimension of 1.96 which corresponds to the expected asymptotic value of 2.0 (classical behavior). Also included are simulations on lattices well above the percolation threshold, $p=0.80(2 \mathrm{~d})$ and $p=0.40$ (3d), where we found that the effective spectral dimension lies in the range $1.33<d_{s}^{\prime}<2.00$. The three-dimensional $p=0.40$ curve is not straight, but 


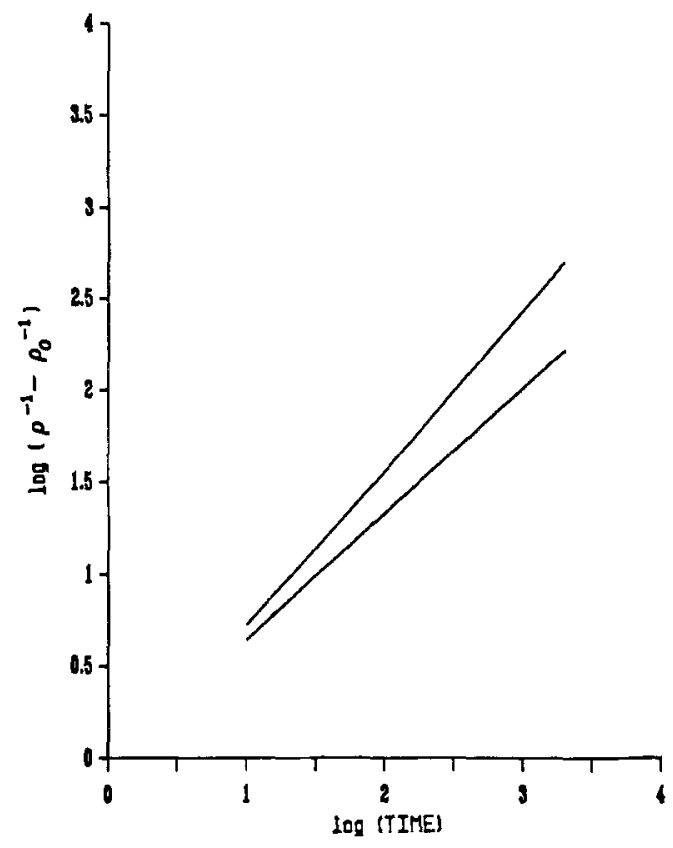

Fig. 3. $\log \left(\langle\rho\rangle^{-1}-\left\langle\rho_{0}\right\rangle^{-1}\right)$ vs. $\log t$ for a two-dimensional square lattice. Top: $p=0.80$, bottom: $p=0.60$. Averages of 100 runs, 2000 steps each, on $200 \times 200$ lattices, with cyclic boundary conditions, $\left\langle\rho_{0}\right\rangle=0.02$. Forced random walks, limited to largest cluster only.

has a visibly increasing slope, typical of a crossover behavior, from fractalto-Euclidean. ${ }^{(16-20)}$

A linear least-squares regression was used to obtain the effective spectral dimension from

$$
\log \left[\langle\rho\rangle^{-1}-\left\langle\rho_{0}\right\rangle^{-1}\right] \propto f^{\prime} \log (t)
$$

where $\langle p\rangle$ is the mean number of walkers over the mean largest cluster size. Results of the regression analysis over the entire time scale and in the asymptotic limit are presented in Table II. The two-dimensional lattices with $p=0.40$ are well below the percolation threshold and an effective spctral dimension of $d_{s}^{\prime}=0.04$ is obtained over the time range studied. This result is not shown.

\subsection{Energetically Disordered Lattices}

Our simulations for reacting random walkers on lattices with energetic disorder exhibit a temperature-dependent fractal-like behavior. Monte Carlo 


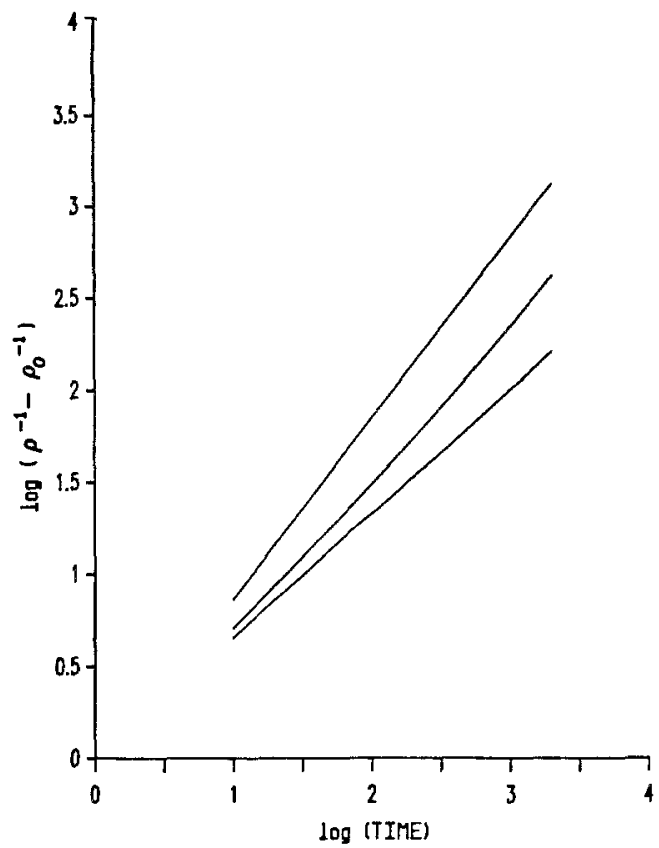

Fig. 4. $\log \left(\langle\rangle^{-1}-\left\langle\rho_{0}\right\rangle^{-1}\right)$ vs. $\log t$ for a three-dimensional simple cubic lattice. Top to bottom: $p=1.00,0.40,0.32$. Averages of 1000 runs, 2000 steps each, on $40 \times 40 \times 40$ lattices, with cyclic boundary conditions, $\left\langle\rho_{0}\right\rangle=0.02$. Forced random walks, limited to largest clusters only.

simulations were performed on these systems for various reduced temperatures $T^{\prime \prime}$ defined above and for the temperature-independent perfect lattices. The temperature-dependent analog of Eq. (7) is

$$
\left\langle\rho^{-1}-\rho_{0}^{-1}\right\rangle=a^{\prime} t^{f^{\prime}\left(T^{\prime \prime}\right)}
$$

Table II. Asymptotic and Overall Percolation Fractal Exponents $f^{\prime}$

\begin{tabular}{lllllll}
\hline & \multicolumn{3}{c}{$2 \mathrm{~d}$} & & \multicolumn{3}{c}{$3 \mathrm{~d}$} \\
\cline { 2 - 3 } \cline { 5 - 7 } \cline { 5 - 7 } & $p=0.60$ & $p=0.80$ & & $p=0.32$ & $p=0.40$ & $p=1.0$ \\
\hline$f^{\prime}{\text { (asymptotic })^{a}}^{\prime}$ & $0.67(4)$ & 0.87 & & $0.68(7)$ & 0.91 & 0.98 \\
$f^{\prime}$ (overall) $^{b}$ & 0.68 & 0.87 & & 0.67 & 0.86 & 0.98 \\
$d_{s} / 2$ & 0.68 & - & & 0.7 & - & 1.0 \\
\hline
\end{tabular}

\footnotetext{
${ }^{a} 516 \rightarrow 2000$ steps.

${ }^{b} 10 \rightarrow 2000$ steps.
} 
and a nonlinear regression is performd to evaluate the exponent $f^{\prime}\left(T^{\prime \prime}\right)$. These results are reported in Table III and $\log -\log$ plots for the square and simple cubic lattices are shown in Figs. 5 and 6, where the straight lines are the fitted curves and the symbols correspond to simulation data. For two dimensions, Fig. 5, for $T^{\prime \prime}=0.2$ and for the perfect lattice, the symbols sit quite nicely on the fitted curves, but at $T^{\prime \prime}=0.1$ the simulation data exhibit a increasing slope when viewed over the entire time scale. In three dimensions, we see a similar behavior. However, the walker in general is less confined for the given reduced temperature. The slightly increasing slope in both two- and three-dimensional disordered lattices may be associated with an effective crossover regime (see binary lattice). Table III presents the results of nonlinear regressions for both the single random walker and the reacting random walkers, Eqs. (4) and (9), for both two and three dimensions. Here we see that the exponent for the single random walker, $f(T)$, and the exponent for the reacting random walkers, $f^{\prime}(T)$, are in reasonably good agreement. These are preliminary results and the error term on each exponent is approximately \pm 0.05 .

\section{CONCLUSIONS}

The conjecture that spectral-fractal exponents apply to binary reactions of random walkers is borne out by simulations on both deterministic fractals (two- and three-dimensional Sierpinski gaskets) and random fractals (twoand three-dimensional percolating clusters). The asymptotic behavior is not

Table III. Energy-Disordered Lattice Effective Fractal Exponents, $f$

\begin{tabular}{|c|c|c|c|c|c|c|}
\hline & \multicolumn{3}{|c|}{$2 \mathrm{~d}$} & \multicolumn{3}{|c|}{$3 \mathrm{~d}$} \\
\hline & $T^{\prime \prime}=0.1$ & $T^{\prime \prime}=0.2$ & $\begin{array}{l}\text { Perfect } \\
\text { latt. }\end{array}$ & $T^{\prime \prime}=0.03$ & $T^{\prime \prime}=0.05$ & $T^{\prime \prime}=0.10$ \\
\hline$f^{\prime}$ (overall) & 0.65 & 0.91 & 0.96 & 0.20 & 0.40 & 0.87 \\
\hline$f$ (overall) & 0.60 & 0.82 & 0.89 & 0.20 & 0.43 & 0.81 \\
\hline$f^{\prime}$ (asymptotic) & 0.71 & 0.9 & 0.98 & 0.22 & 0.46 & 0.92 \\
\hline$f$ (asymptotic) & 0.85 & 0.91 & 0.93 & 0.28 & 0.36 & 0.89 \\
\hline \multicolumn{7}{|c|}{$\begin{array}{l}f^{\prime}(2 \mathrm{~d} \text { asymptotic limit }): \rightarrow \text { times of } 0 ; 3000 \rightarrow 5000 \text { except for } T^{\prime \prime}=0.2 \text {, } \\
\text { which uses } 0 ; 2000 \rightarrow 4000\end{array}$} \\
\hline \multicolumn{7}{|c|}{$f^{\prime}(3 \mathrm{~d}$ asymptotic limit $): \rightarrow$ times $0 ; 1500 \rightarrow 2000$} \\
\hline \multicolumn{7}{|c|}{$\begin{array}{l}f \text { (asymptotic limit): } 0 ; 50,000 \rightarrow 100,000(2 \mathrm{~d}) 0 ; 1500 \rightarrow 2000(3 \mathrm{~d}) \\
\quad \text { overall: } 0 \rightarrow 4000(2 \mathrm{~d}) 0 \rightarrow 2000(3 \mathrm{~d}) .\end{array}$} \\
\hline
\end{tabular}




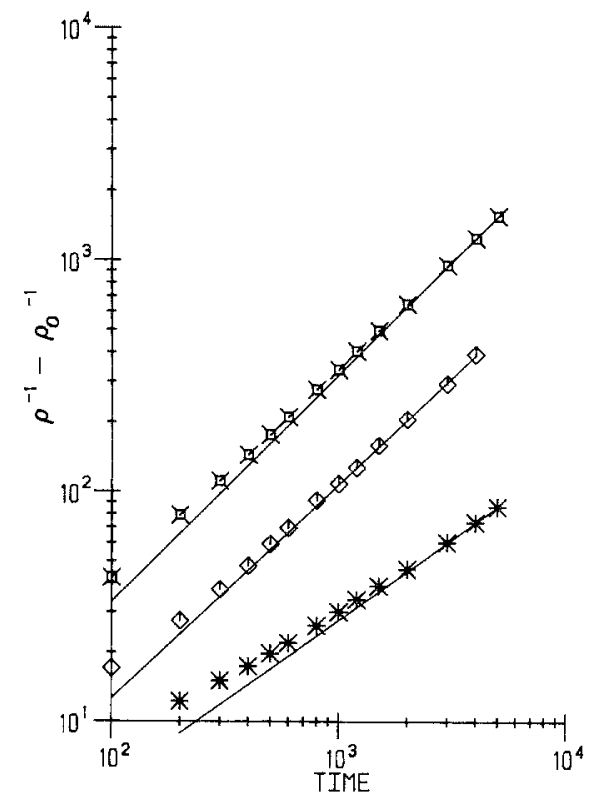

Fig. 5. $\log \left\langle\rho^{-1}-\rho_{0}^{-1}\right\rangle$ vs. $\log t$ for a two-dimensional perfect square lattice (top curve) and for square lattices with energetic disorder $T^{\prime \prime}=0.2$ (middle curve) and $T^{\prime \prime}=0.1$ (bottom curve). In all cases $\rho_{0}=0.002$. Simulation results (symbols) and fitted curves (long time limits) are shown.

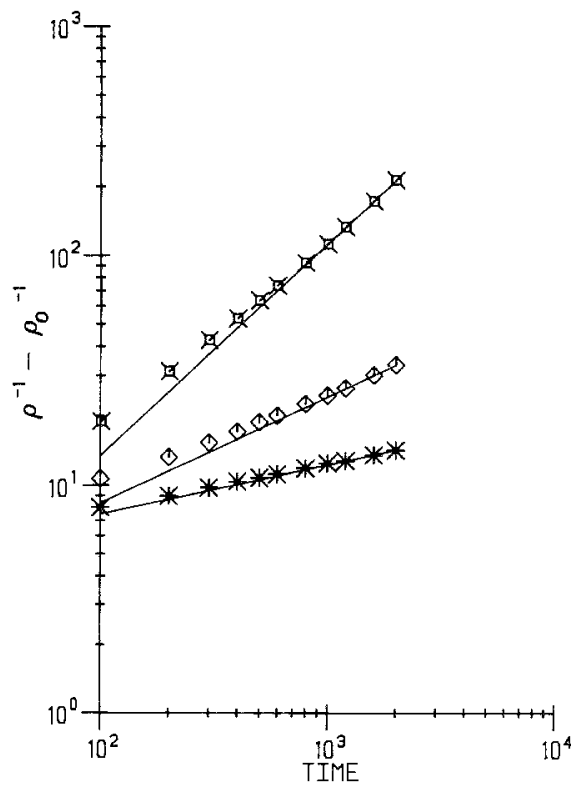

Fig. 6. $\log \left\langle\rho^{-1}-\rho_{0}^{-1}\right\rangle$ vs. $\log t$ for a three-dimensional energetically disordered cubic lattice. Top to bottom: $T^{\prime \prime}=0.1,0.05,0.03$. Simulation results (symbols) are averages of ten runs on $25 \times 25 \times 25$ lattices with $\rho_{0}=0.1$. The fitted curves are obtained from the nonlinear regressions (long time limits). 
sensitive to perturbations away from a uniformly random initial distribution. These findings may be of much relevance to reactions in microscopically heterogenous media, as shown by our simulations of reacting walkers on two- and three-dimensional energetically disordered lattices.

\section{NOTE ADDED IN PROOF}

For the steady state binary reaction we find that the average annihilation rate is directly proportional to $\rho^{x}$ where $x=1+f^{-1}$ in the low density limit. Simulations on the planar Sierpinski gasket give $x=2.44$ $\left(1+f^{-1}=2.45\right)$; on a perfect cubic lattice $x=2.01\left(1+f^{-1}=2\right)$, i.e., the classical result.

\section{REFERENCES}

1. S. Alexander and R. Orbach, J. Phys. (Paris) Lett. 43:L625 (1982).

2. R. Rammal and G. Toulouse, J. Phys. (Paris) Lett. 44:L13 (1983).

3. S. Alexander, in Percolation Structures and Processes, G. Deutscher, R. Zallen, and J. Adler, eds., Annals of the Israel Physical Society, Jerusalem (1983), p. 149.

4. B. B. Mandelbrot, The Fractal Geometry of Nature (W. H. Freeman, San Francisco, 1983).

5. P. W. Klymko and R. Kopelman, J. Phys. Chem. 87:4565 (1983).

6. P. Evesque, J. Phys. (Paris) 44:1217 (1983).

7. K. Kang and S. Redner, Phys. Rev. Lett. 52:955 (1984).

8. P. G. de Gennes, C. R. Acad. Sci. Ser. A 296:881 (1983).

9. D. Toussaint and F. Wilczek, J. Chem. Phys. 78:2642 (1983).

10. D. C. Torney, J. Chem. Phys. 79:3606 (1983).

11. D. C. Torney and H. M. McConnell, J. Phys. Chem. 87, 1441 (1983).

12. D. C. Torney and H. M. McConnell, Proc. R. Soc. London Ser. A 387:147 (1983).

13. R. M. Noyes, Prog. React. Kinetics 1:128 (1961).

14. E. W. Montroll, J. Chem. Phys. 14:202 (1946).

15. R. Kopelman, P. W. Klymko, J. S. Newhouse, and L. Anacker, Phys. Rev. B, in press.

16. Y. Gefen, A. Aharony, and S. Alexander, Phys. Rev. Lett. 50:77 (1983).

17. I. Webman, Phys. Rev. Lett. 52:220 (1984).

18. P. Argyrakis and R. Kopelman, Phys. Rev. B 29:511 (1984).

19. S. Havlin, D. Ben-Avraham, and H. Sompolinski, Phys. Rev. A 27:1730 (1983).

20. P. Argyrakis and R. Kopelman, J. Chem. Phys. 81:1015 (1984).

21. P. Argyrakis, L. W. Anacker, and R. Kopelman, J. Stat. Phys. 36:551 (1984).

22. Daniel Fox, A Set of Computer Routines for the Generation of Pseudo-Random Numbers, Technical Report 4, Statistical Res. Lab., University of Michigan, February 1977.

23. B. A. Weichmann and I. D. Hill, Appl. Stat. 31:188 (1982).

24. C. D. Mitescu and J. Roussenq, in Percolation Structures and Processes, G. Deutscher, R. Zallen, and J. Adler, eds., Annals of the Israel Physical Society, Jerusalem (1983), p. 81 .

25. A. Blumen, J. Klafter, and G. Zumofen, Phys. Rev. B 28:6112 (1983); 27:3429 (1983).

26. J. C. Angles D'Auriac, A. Benoit and R. Rammal, J. Phys. A 16:4039 (1983). 